\title{
Key factors impacting performance of a salinity gradient solar pond exposed to Mediterranean climate
}

\author{
Mehdi Amouei Torkmahalleh a,b,*, Mohammad Askari ${ }^{\mathrm{c}}$, Soudabeh Gorjinezhad ${ }^{\mathrm{b}}$, Dilem Eroğlu ${ }^{\mathrm{b}}$, \\ Madina Obaidullah ${ }^{\mathrm{d}}$, Abdullrahman Rajab Habib ${ }^{\mathrm{e}}$, Sevgi Godelek ${ }^{\mathrm{b}}$, Sultan Kadyrov ${ }^{\mathrm{e}}$, Orhun Kahraman ${ }^{\mathrm{e}}$, \\ Negar Zare Pakzad ${ }^{\mathrm{f}}$, Goodarz Ahmadi ${ }^{\mathrm{g}}$ \\ ${ }^{a}$ Chemical and Aerosol Research Team, School of Engineering, Nazarbayev University, Astana, 010000, Kazakhstan \\ ${ }^{\mathrm{b}}$ Chemical Engineering Program, Middle East Technical University Northern Cyprus Campus, Guzelyurt, Mersin 10, Turkey \\ ${ }^{\mathrm{c}}$ Mechanical Engineering Department, Bilkent University, Ankara, 6800, Turkey \\ ${ }^{\mathrm{d}}$ Sustainable Environment and Energy Systems Program, Middle East Technical University Northern Cyprus Campus, Guzelyurt, Mersin 10, Turkey \\ ${ }^{\text {e } C h e m i c a l ~ E n g i n e e r i n g ~ D e p a r t m e n t, ~ M i d d l e ~ E a s t ~ T e c h n i c a l ~ U n i v e r s i t y, ~ A n k a r a, ~ 06531, ~ T u r k e y ~}$ \\ ${ }^{\mathrm{f}}$ Mechanical Engineering Program, Middle East Technical University Northern Cyprus Campus, Guzelyurt, Mersin 10, Turkey \\ ${ }^{\mathrm{g}}$ Department of Mechanical Engineering, Clarkson University, Potsdam, NY, 13699-5725, USA
}

\section{A R T I C L E I N F O}

\section{Article history:}

Received 15 November 2015

Received in revised form 16 October 2016

Accepted 18 December 2016

Available online 30 December 2016

\section{Keywords:}

Solar pond

Salinity

Mediterranean climate

UCZ

$\mathrm{NCZ}$

LCZ

\begin{abstract}
A B S T R A C T
Solar ponds are low cost pools of brine solutions with integrated storage zones that harvest incident solar energy and store it as thermal energy. The current study examined the performance of a salinity gradient solar pond under the Mediterranean climatic condition for ten consecutive months of operation, from October 8, 2014 to July 31, 2015. The presented results are based on the experimental data of a smallscale circular pond, $61 \mathrm{~cm}$ in diameter a height of $55 \mathrm{~cm}$, constructed and operated at Middle East Technical University, Northern Cyprus Campus (METUNCC). The study showed the necessity of regular surface washing and having excess undissolved salt at the lower convective zone (LCZ) to maintain the pond stability. The variations in the temperature of the non-convective (NCZ) and lower convective zones (LCZ) are found to be a function of both ambient temperature and solar irradiation (insolation). The variation of the overall pond's temperature strongly follows the changes in ambient temperature while solar insolation directly affects the increase in temperature gradient by depth. During the period of this study, the pond reached the highest average temperature of $48^{\circ} \mathrm{C}$ in July 2015 while the average ambient temperature for this month was $30^{\circ} \mathrm{C}$.
\end{abstract}

๑) 2016 Elsevier Ltd. All rights reserved.

\section{Introduction}

Renewable energy sources have been gaining greater importance in the recent years. It is crucial to develop devices and processes to supply energy from non-polluting and renewable energy sources for sustainable developments. The solar pond is an example of such devices that collects solar energy and stores it as thermal energy for a long duration. The first reference to a naturally occurring brine solar pond is attributed to Kalecsinsky in 1902 with his observations of temperatures about $70^{\circ} \mathrm{C}$ at a depth of $1.3 \mathrm{~m}$ in the Medve Lake in Transylvania (El-Sebaii et al., 2011). Kalecsinsky was the first to record and address the correlation between temperature and salt concentration profiles in ponds.

\footnotetext{
* Corresponding author at: Chemical and Aerosol Research Team, School of Engineering, Nazarbayev University, Astana 010000, Kazakhstan.

E-mail address: mehdi.torkmahalleh@nu.edu.kz (M. Amouei Torkmahalleh).
}

The corresponding theoretical was performed by Weinberger (1964) where an understanding the thermophysical behavior of solar ponds was presented. This was followed by making the first large-scale artificial solar pond for thermal energy storage by Tabor and Matz (1965). Most experimental studies on practical utilization of artificial solar ponds were started mainly around mid1970s (Norton, 1992). These earlier studies indicated that the temperature in solar ponds may reach up to $70-80{ }^{\circ} \mathrm{C}$ implying that the thermal energy from solar ponds can be useful for applications with low-grade energy demands (Sukhatme and Nayak, 2008). One of the most important advantages of solar ponds over other renewable energy sources, such as solar collectors, is their lower investment cost (Sukhatme and Nayak, 2008). Solar ponds are environmentally friendly, and can be used for heating and/or electricity generation. The heat obtained from a solar pond can be converted into electric power even at low temperature values (Bernad et al., 2013). In this regard, organic Rankine cycle engines are 
typically operated using the temperature difference in a solar pond. For applications where the organic fluid fails to operate because of a low temperature difference, thermoelectric generator could be a good candidate to replace ORC engines for power generation (Singh et al., 2012).

The principle of solar ponds is quite simple. In ordinary ponds, the solar radiation reaching the pond is absorbed by water which causes the lower level water to heat up. The heated water has lower density, and thus rises to the pond surface due to natural convection and loses its thermal energy to the atmosphere. In solar ponds, the natural convection is inhibited in order to retain the absorbed heat at the bottom of the pond. The suppression of buoyancy-driven natural convection is done by creating a salinity gradient in the pond and making the water at the bottom denser. Salinity gradient solar ponds typically consist of three layers. The first layer, known as the upper convective zone (UCZ), is located at the top of the pond, and contains the least salinity level. The second layer, whose salinity level increases with depth, is called nonconvective zone (NCZ). This layer suppresses the convection and acts as an insulator to prevent the heat from escaping to the UCZ and for maintaining a high temperature at deeper depth. The last layer, made of a saturated salt solution, is responsible for energy storage and is known as the lower convective zone (LCZ) (Zangrando, 1980; Tabor and Weinberger, 1981). The performance of a solar pond decreases with the increase of evaporation rate and decreasing salinity gradient (Ouni et al., 1998).

There have been a number of small-scale experimental studies carried out on salinity gradient solar ponds in different regions of the world under different climatic conditions. Bernad et al. (2013) performed an experimental work to examine the performance of a $50 \mathrm{~m}^{2}$ solar pond in Spain. According to their findings, the small-scale pond reached a maximum temperature of $75^{\circ} \mathrm{C}$ in summer with about $16 \%$ of incident radiation available for extraction. Dah et al. (2005) conducted a study on a laboratory scale pond in Tunisia and analyzed the evolution of temperature and salinity profiles in the pond during one month of operation. They attained a maximum temperature of $45^{\circ} \mathrm{C}$ having a temperature difference of $23^{\circ} \mathrm{C}$ between the bottom and the surface of the pond. Karim et al. (2010) performed an experimental study to analyze and maintain the stability of solar ponds in Tunisia. Using the MATLAB model developed by Jaefarzadeh (2004), Sakhrieh and Al-Salaymeh (2013) investigated the performance of a solar pond under Jordanian climate conditions during two weeks of the operation. Their result indicated that temperature at the LCZ correlated well with the ambient temperature variations. They achieved a maximum temperature of $47^{\circ} \mathrm{C}$ when the ambient temperature was approximately $30^{\circ} \mathrm{C}$. Nie et al. (2011) constructed a large-scale shallow salt gradient solar pond using the natural brine of a local salt lake in the Tibet plateau. They observed that the LCZ temperature varied from $20^{\circ} \mathrm{C}$ to $40{ }^{\circ} \mathrm{C}$ over one year. El-Sebaii et al. (2013) investigated the thermal performance of a shallow salt gradient solar pond under open and closed modes of heat extraction, under Egyptian weather conditions. They concluded that their constructed pond could be used as a heat source for most domestic and lowgrade energy demand industrial applications all year-round. Karakilcik et al. (2006) investigated the experimental and theoretical temperature distributions and amount of heat losses within a solar pond in Turkey. They found that the total heat losses from the surface of the pond accounted for more than $85 \%$ of the losses, while the losses from the side walls and ground were far less.

Small-scale solar ponds can be useful for investigating the important factors affecting the performance of large-scale solar ponds (Suárez et al., 2014). In particular, small-scale solar ponds can be operated for a longer period because of the lower operation and maintenance cost, and under more controlled conditions compared to large-scale solar ponds. Performance of solar ponds is greatly influenced by climatic factors including the ambient temperature, radiation, wind velocity and relative humidity. The significance of these factors varies with the regions making it imperative to study the solar pond performance under the climate of the different regions of the world. While there have been a number of studies on salt gradient solar ponds in different countries, the performance of a solar pond under the Mediterranean climate (Northern Cyprus), has not been yet well evaluated. Additionally, none of the previous experimental studies fully investigated on the impact of the individual aforementioned factors on the performance of a solar pond in a single study. Northern Cyprus experiences the Mediterranean climate enriched in both solar and wind energy together with relatively high humidity and ambient temperature.

The present study was designed to investigating the performance of a salinity gradient solar pond in a region with high levels of solar radiation and wind energy. Another objective of this study was to provide an understanding of the impact of ambient temperature and solar intensity on the performance and temperature variation in the pond. Furthermore, this study provided a comprehensive set of metrological data including ambient temperature, insolation, wind velocity and relative humidity with a daily time resolution for over one year operation of solar pond. These data would be very useful in understanding the dynamics of solar ponds for long-term operations and for model validation purposes. In fact, the previous experimental investigations rarely provided such all the needed information for modeling studies. Another novelty of the present study was to investigate the practical considerations for improving the salt stability which is a key for the proper operation of the large-scale salinity gradient solar ponds.

A small-scale salinity gradient solar pond was constructed and operated at Middle East Technical University, Northern Cyprus Campus (METUNCC) located in Guzelyurt, Northern Cyprus. The pond has been operating since October 8, 2014 and experimental results have been continually recorded. This study presents the experimental and meteorological data from October 8, 2014 to July 31, 2015 for the operation of the METUNCC solar pond. The performance study of the current solar pond together with the meteorological data can provide the needed information for validating a computational model that can be used for evaluating the performance of large- scale solar ponds under various climatic conditions.

\section{Materials and methods}

A cylindrical pond $(61 \mathrm{~cm}$ diameter, $55 \mathrm{~cm}$ height and $1.2 \mathrm{~cm}$ thickness) was constructed and installed at METUNCC. The pond had three zones, the UCZ (15 L), NCZ (45 L) and LCZ (75 L). Table salt was utilized to make salinity solutions. The LCZ was composed of a saturated (C) salt solution (approximately $280 \mathrm{~g} / \mathrm{l}$ ). The NCZ was made of three equally divided sub-layers (each one $15 \mathrm{~L}$ ) with $3 \mathrm{C} / 4, \mathrm{C} / 2$ and $\mathrm{C} / 4$ concentrations. The UCZ contained fresh water. Six thermometers ( 2 for each layer) were installed on the pond to monitor the daily temperature variations at 9 a. m., 1 p.m., 5 p.m., and 10 p.m. Temperature recording at other time of the day were performed as needed. The pond was equipped with three sampling valves to withdraw samples from each layer to monitor the salt concentrations. The middle valve was located at the middle of the NCZ. Three inlet ports were also installed on the pond to add proper amount of solutions to each zone during the experiments to compensate for evaporation and sampling losses as well as for surface washing. The inner surfaces of the pond were painted black. The bottom and sides of the pond were initially insulated with a $2.5 \mathrm{~cm}$ thick thermal insulator, and the insulation layers were thickened to $5.0 \mathrm{~cm}$ after the first three months of the operation to minimize the heat loss from the walls. 
Moreover, the pond was placed on a $10 \mathrm{~cm}$ thick polyurethane insulator foam to further minimize the heat loss to the ground. Experimental investigations showed no salinity gradient disturbance because of the insulation improvement. Figs. $1 \mathrm{a}$ and $1 \mathrm{~b}$ respectively, show a schematic representation and actual photo of the experimental setup.

Excess (undissolved) salt was added to the bottom of the pond to ensure saturation of the bottom layer. The ambient temperature was monitored every 10 minutes using a thermometer sensor (Galltec 1/5, Germany). Solar radiation was monitored every 10 minutes using a sun-tracking device (Kipp and Zonen, France), placed next to the solar pond, along with a Pyranometer to record Global Horizontal Insolation (GHI). Wind velocity was measured at $30,40,50$ and $60 \mathrm{~m}$ above the ground using wind sensors (Thies- Germany). The wind velocity was then corrected to find the velocity, $15 \mathrm{~cm}$ above the pond surface using Johnson (1999), Sadati et al. (2015).

$\frac{U_{1}}{U_{2}}=\frac{\ln \left(\frac{Z_{1}}{Z_{0}}\right)}{\ln \left(\frac{Z_{2}}{Z_{0}}\right)}$

where $U_{1}$ and $U_{2}(\mathrm{~m} / \mathrm{s})$ are the velocity values, respectively, at elevations $Z_{1}$ and $Z_{2}(\mathrm{~m})$ from the ground. $Z_{0}$ is the roughness length $(\mathrm{m})$. To estimate $Z_{0}$ for the current study using Eq. (1), and the measured wind velocity values at $30,40,50$ and $60 \mathrm{~m}$ were used. The average roughness length was estimated to be $0.61 \mathrm{~m}$. The wind velocity values at $30 \mathrm{~m}$ and Eq. (1) are then used to find the wind velocity at $70 \mathrm{~cm}$ above the ground that is about $15 \mathrm{~cm}$ above the pond surface. The relative humidity $(\mathrm{RH})$ was monitored using a RH sensor (Galltec, KPC, Germany).

To prevent accumulation of diffused salt in the UCZ as well as to compensate for evaporation and sampling losses in the UCZ, the surface of the pond was regularly washed every 3 days. This washing cycle was established after three weeks of the operation when significant salt concentration was observed at the UCZ (Fig. 3). This routine washing maintained the initial salinity gradient and volume of the UCZ. In the summer, surface washing was done every 2 days or even daily depending on the evaporation rate. To wash the surface, most of the UCZ water was emptied first such that about $6 \mathrm{~L}$ of UCZ water were left. The amount of removed water from the UCZ varied with the extent of rain and evaporation. Then, $9 \mathrm{~L}$ of fresh tap water were added at a very slow flow rate to maintain the initial volume $(15 \mathrm{~L})$ of the UCZ. Prior to surface washing, a

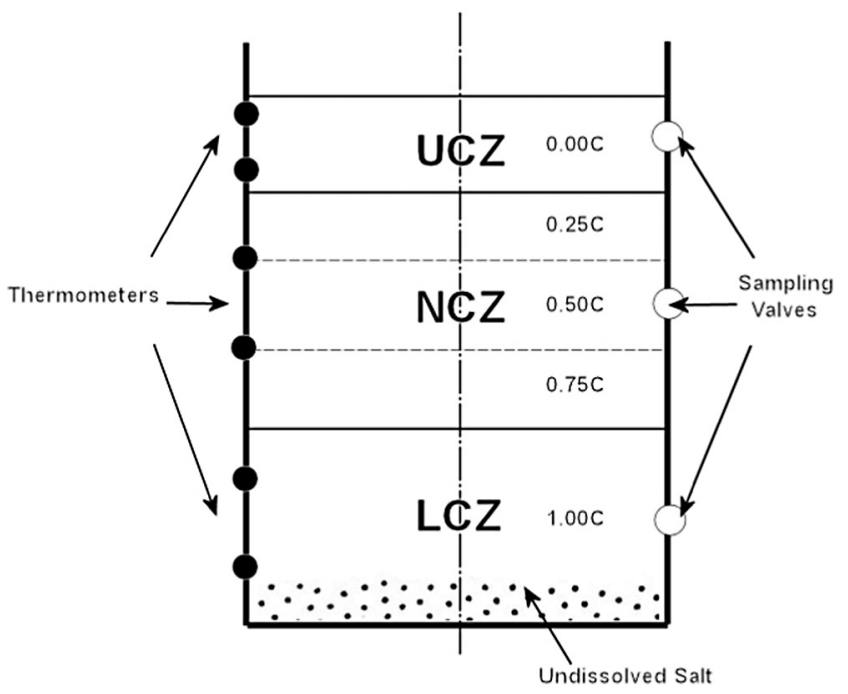

Fig. 1a. Schematic representation of the experimental setup - C represents saturated salt solution level. sample was taken from UCZ to monitor the salt concentration. In addition, approximately 1 hour after surface washing a sample was withdrawn from the pond to ensure availability of fresh water at the UCZ. Due to routine surface washing, the salt concentration of UCZ was maintained at approximately its initial level.

To monitor the salt concentrations, samples $(20 \mathrm{ml})$ were taken from each layer through the connected valves, every three days. Each sample solution was then diluted 25 times into $250 \mathrm{ml}$ flasks, separately. The conductivity of the diluted solutions was measured using a calibrated conductivity meter (Multi 3420, WTW, Germany). Then, the salt concentration of each layer was measured using a calibration curve. Fig. 2 shows the conductivityconcentration calibration curve with a coefficient of determination of $\left(R^{2}\right) 0.9999$.

To compensate for the sampling losses of the NCZ and LCZ, $100 \mathrm{ml}$ of salt solutions with the same salinity level as the initial $\mathrm{NCZ}$ and LCZ concentrations were added every 2 weeks, to both $\mathrm{NCZ}$ and LCZ.

To calculate the average daily temperature of the pond for each layer as well as the average daily ambient temperature, an average was taken over the four temperature readings (both for the pond and ambient temperatures) in a day. Daily GHI value was calculated as the cumulative sum of the GHI over 24 hours for a day of interest. The monthly average of each quantity was calculated as the average of the corresponding daily data.

\section{Results and discussion}

Fig. 3 shows the salinity concentration of each layer during the pond operation. The first data point for each layer corresponds to the initial concentration of each layer. As of day 18, the surface of the pond was washed with fresh water roughly every three days to control the salinity level. Salinity disturbance due to the high rate of salt diffusion toward the UCZ was observed during the first eighteen days of the operation when surface washing was not done intentionally. The experiment clearly showed the necessity of regular surface washing to prevent instability due to salinity disturbance. During this period, LCZ and NCZ concentrations remained nearly unchanged while the UCZ concentration increased. This observation implies that the dissolution of the excess salt into the LCZ compensated for the diffused salt and, therefore, maintained the saturated salinity at the LCZ. Another evidence for this conclusion was observed at about day 240 when the LCZ and NCZ concentrations started decreasing while UCZ concentration increased suggesting salt diffusion from bottom of the pond to the top. In fact, the surface washing was stopped from day 234 to day 244 due to unforeseen circumstances. Although as of day 244 surface washing restarted, and were performed every three days, the salt concentration at UCZ remained higher than the normal value. This was also the reason that during days 244-257, disturbances in the concentration profiles at different pond layers were observed. It appears that the rise of temperature during hot seasons not only affected the evaporation rate but also increased the salt diffusion rate which in return required a more regular surface washing cycle. Nonetheless, starting from day 257, surface washing was performed daily such that the UCZ concentration remained at the normal level throughout the rest of the campaign. The NCZ and LCZ concentrations were then adjusted back to the normal condition implying dissolution of the excess salt into the LCZ. To the best knowledge of the authors, these experimental observations were not explicitly discussed before in the literature. An improper washing event with a fast addition of UCZ water during surface washing, is also indicated in Fig. 3. In fact, this improper washing caused a bit of mixing between the UCZ and NCZ, which resulted in the drop of concentration in the NCZ. By adding brine to the $\mathrm{NCZ}$, the concentration was adjusted back to the initial 


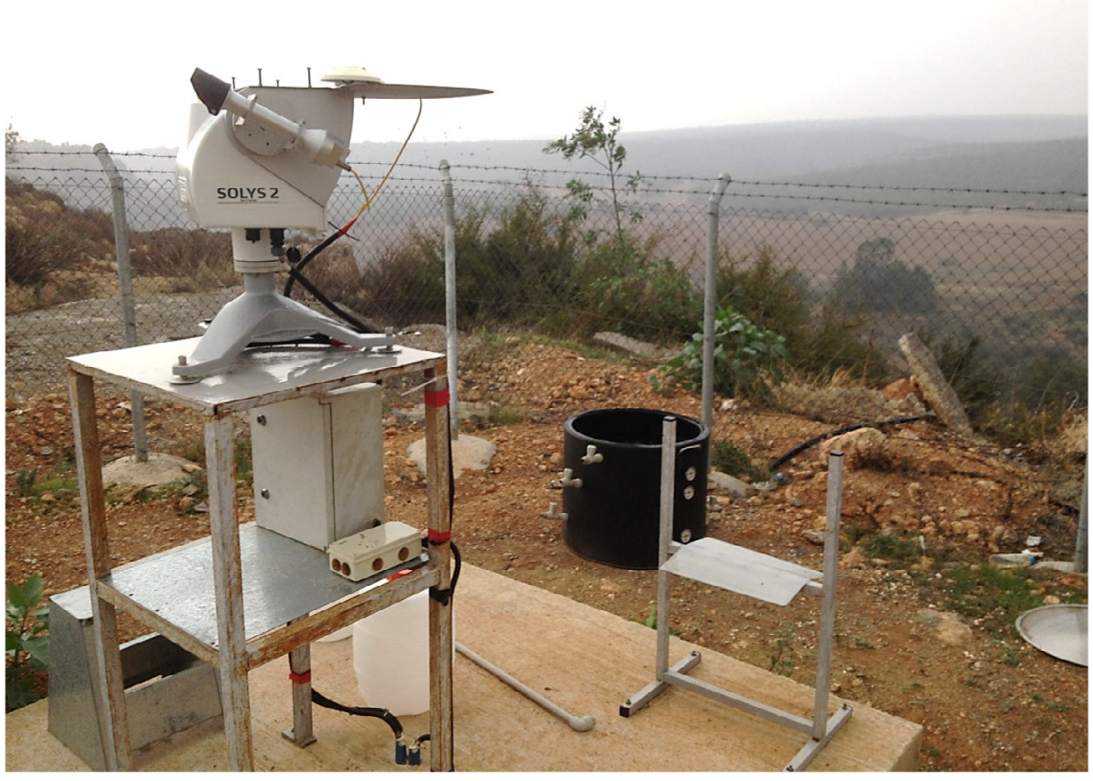

Fig. 1b. The experimental setup.

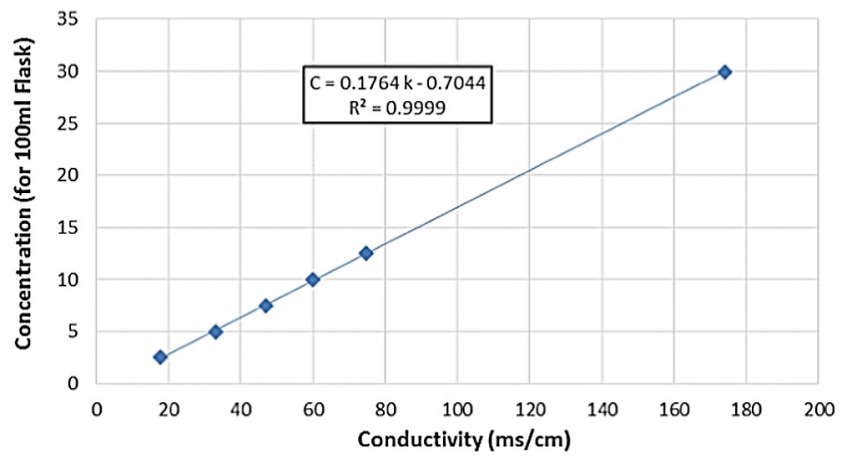

Fig. 2. Conductivity-concentration calibration curve.

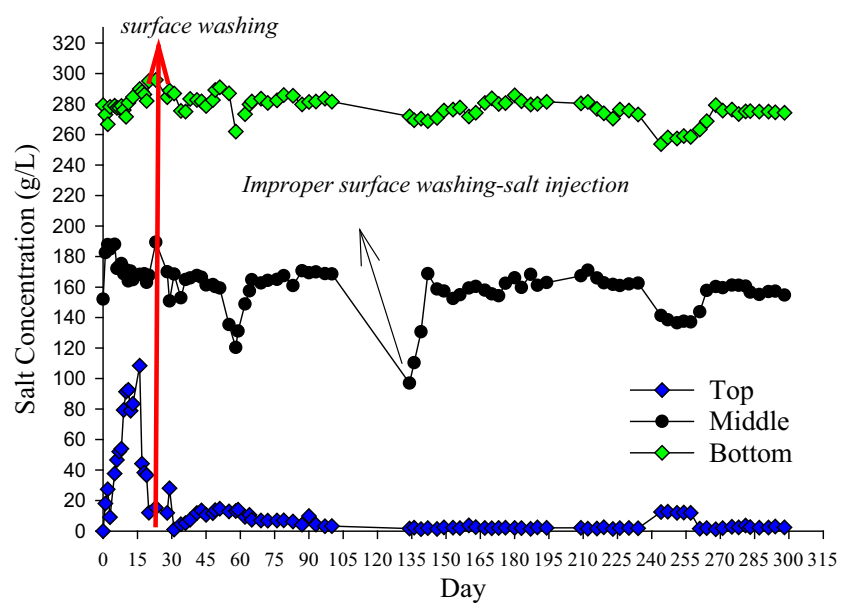

Fig. 3. Average salt concentration for each layer.
NCZ concentration. Overall, Fig. 3 confirms the stability of the salt concentration gradient during the operation of the solar pond.

The ambient temperature variations together with average (four measurements) daily temperature values for each layer are shown in Figs. 4a-4d. Since the top layer of the pond was open to the atmosphere, the temperature of the UCZ (Fig. 4b) was always nearly the same as the ambient temperature (Fig. 4a). The temperature increased with depth such that the LCZ (Fig. 4d) showed the highest temperature compared to the NCZ (Fig. 4c) and UCZ. This increasing trend of temperature with depth was observed for the entire operation period implying that the pond functioned properly. At the start of the operation in October 2014, the LCZ temperature was $35^{\circ} \mathrm{C}$ (Fig. 4d) when the average ambient temperature was $26^{\circ} \mathrm{C}$ (Fig. 4a). As the cold season started, the temperature of the three layers decreased which resulted in a minimum temperature of $5.5,8.25$ and $9.75^{\circ} \mathrm{C}$, respectively, for $\mathrm{UCZ}, \mathrm{NCZ}$ and LCZ after 93 days of the operation (January 8, 2015). These mini-

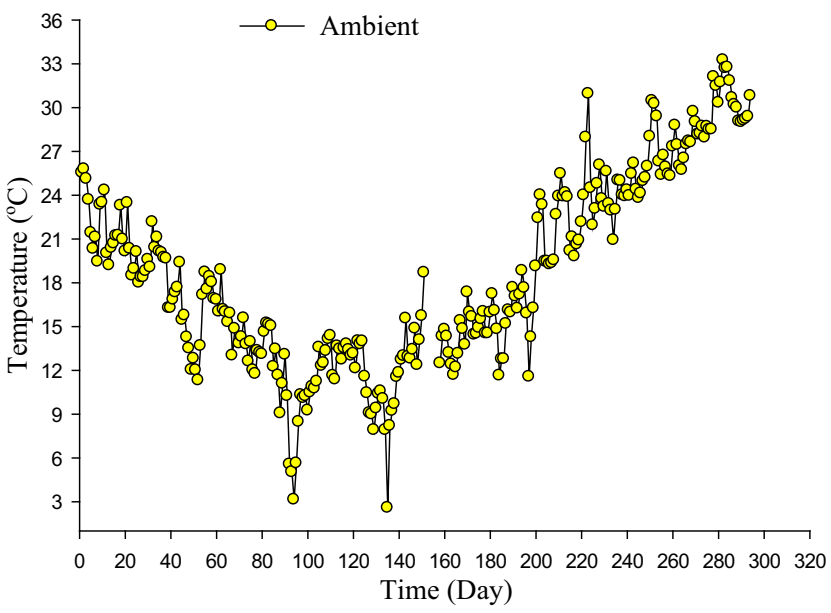

Fig. 4a. Average daily ambient temperature. 


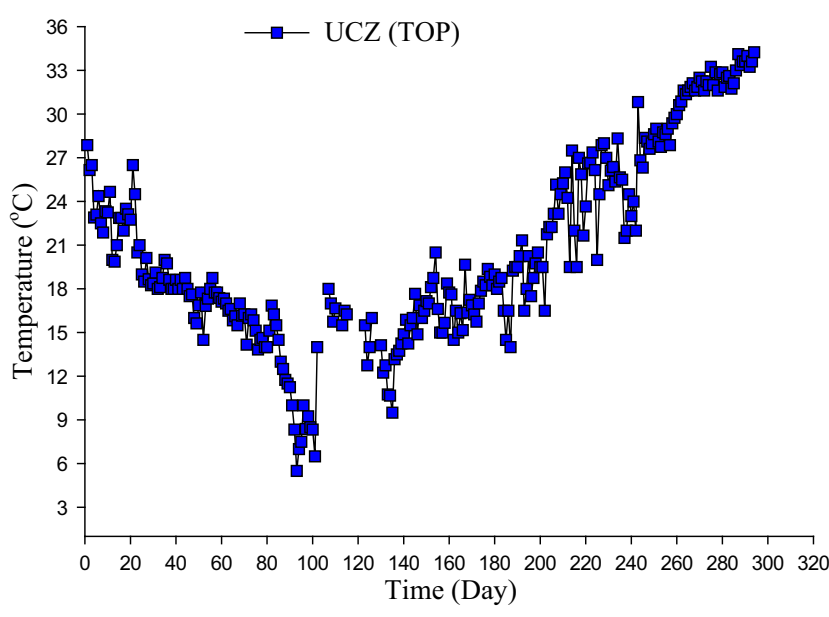

Fig. 4b. Average daily temperature of UCZ (top layer).

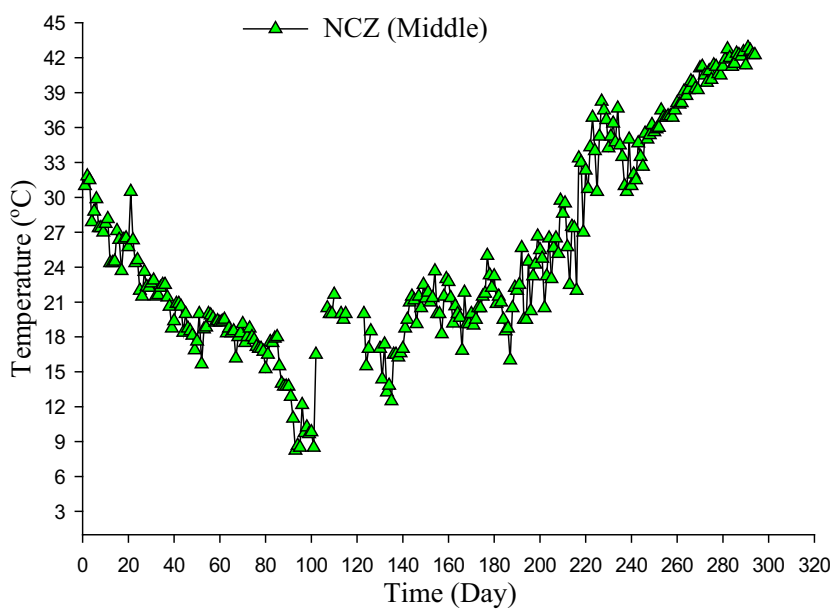

Fig. 4c. Average daily temperature of NCZ (middle layer).

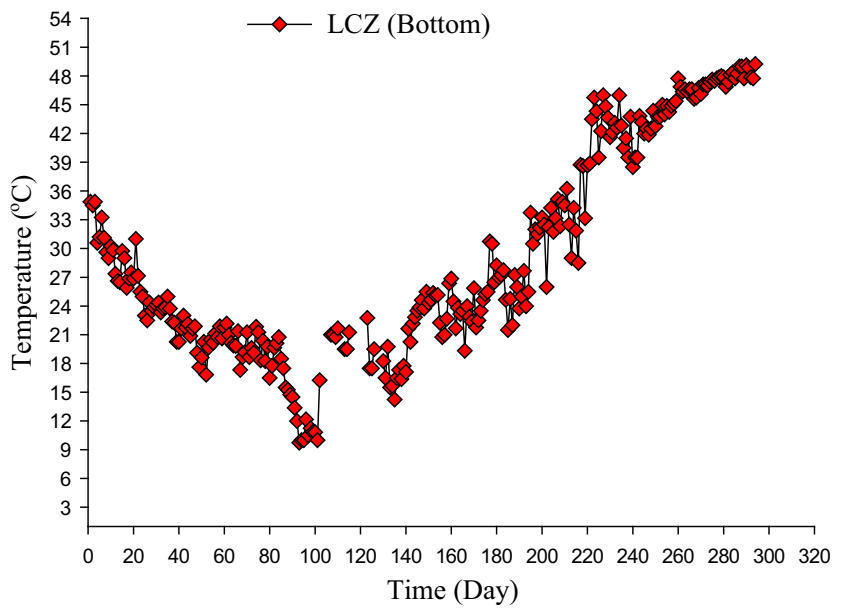

Fig. 4d. Average daily temperature of LCZ (bottom layer).

mum values were associated with a minimum in the ambient temperature. Two subsequent major minimum days were observed approximately on day 135 (early February) and 241 (early June). The second minimum was also correlated with a drop in ambient temperature indicating the important impact of ambient tempera- ture on the performance of this small solar pond. Furthermore, Fig. 3 showed a salinity gradient disturbance of NCZ on day 135 which could be another reason for the observed temperature drop. After the second minimum, when the warm season commenced, a trend of increasing temperature was observed for all three layers. An interesting observation was made in the pond performance around day 241 (third minimum), corresponding to the noticeable drop in the temperatures of all layers despite the increase in ambient temperature. This implied the presence of another key factor affecting the solar pond performance. Reviewing the daily insolation values shown in Fig. 6 revealed no significant changes in insolation values during day 237 to day 243 . Thus, it is most likely that the salinity gradient disturbance occurred on day 241 (Fig. 3), was responsible for the temperature disturbance of the solar pond due to convective heat losses. This further shows the importance of maintaining the salinity gradient ensures higher performance of the pond. This temperature disturbance decreased the rate of increase in pond temperature implying that it might had been possible to reach higher temperature values during the last month of operation (July) if the disturbance would have been avoided. The maximum average daily LCZ temperature was recorded to be $49{ }^{\circ} \mathrm{C}$ on day 295 (July 29, 2015). Average daily temperature variations of each layer (Figs. 4b-4d) followed almost the same trend as the average daily ambient temperature (Fig. 4a) demonstrating the key influence of ambient temperature on the dynamics of the solar pond.

Figs. 5-7 present respectively, the daily temperature differences between the LCZ and UCZ, between the LCZ and NCZ, and daily GHI variations. These figures show strong correlations between the temperature difference and GHI variations. That is, as GHI reduced the difference between the temperature of the LCZ and UCZ as well as LCZ and NCZ decreased. On some days, the NCZ temperature was equal to the LCZ temperature. As of day 90 (January 6, 2015) to approximately day 240 , increasing trends in temperature differences with increased GHI were observed in both figures. From day 1 (October 8, 2014) to day 50 (November 27, 2014) the temperature difference variations plotted in Figs. 5 and 6 were well correlated with GHI variations. The temperature variations from day 50 (November 27, 2014) to day 75 (December 22, 2014) however, did not follow the GHI variations in this period of the operation. As seen in Figs. 4 and 5, from day 50 to day 65 the temperature differences increased while GHI decreased. Additionally, from day 65 (December 12, 2014) to day 75 (December 22, 2014) the temperature differences decreased while the GHI remained roughly constant. The temperature difference variations

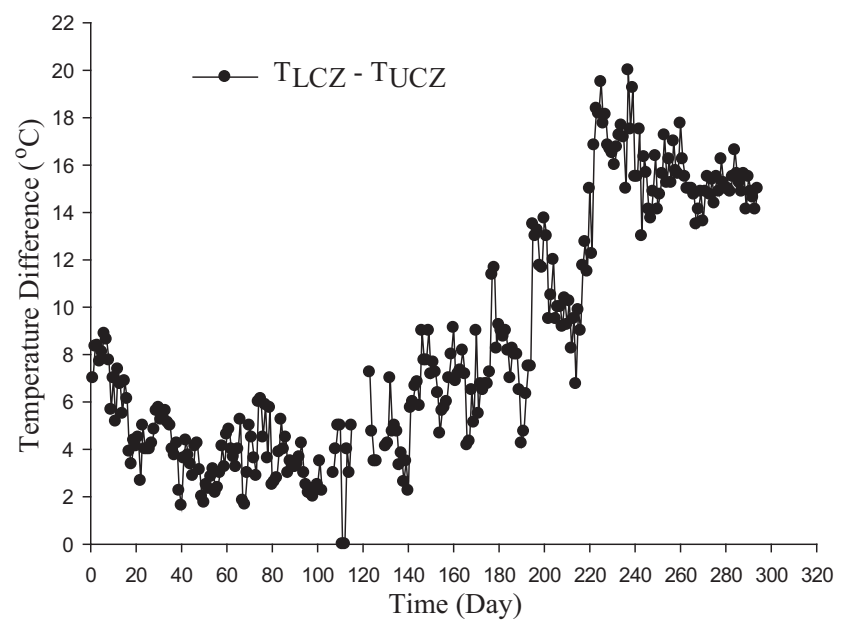

Fig. 5. Average daily temperature difference between LCZ $\left(T_{L C Z}\right)$ and UCZ ( $T_{U C Z}$ ) 


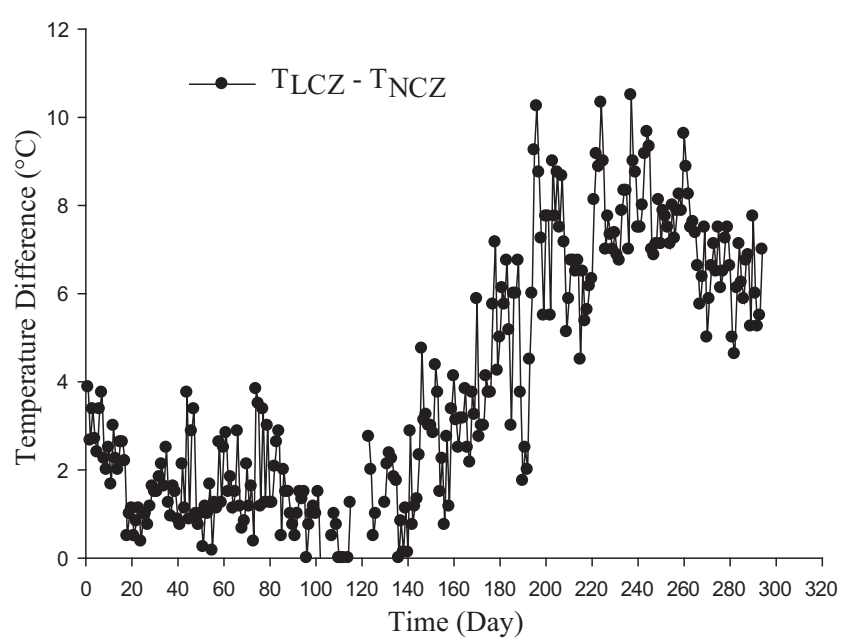

Fig. 6. Average daily temperature difference between LCZ $\left(T_{L C Z}\right)$ and NCZ $\left(T_{N C Z}\right)$.

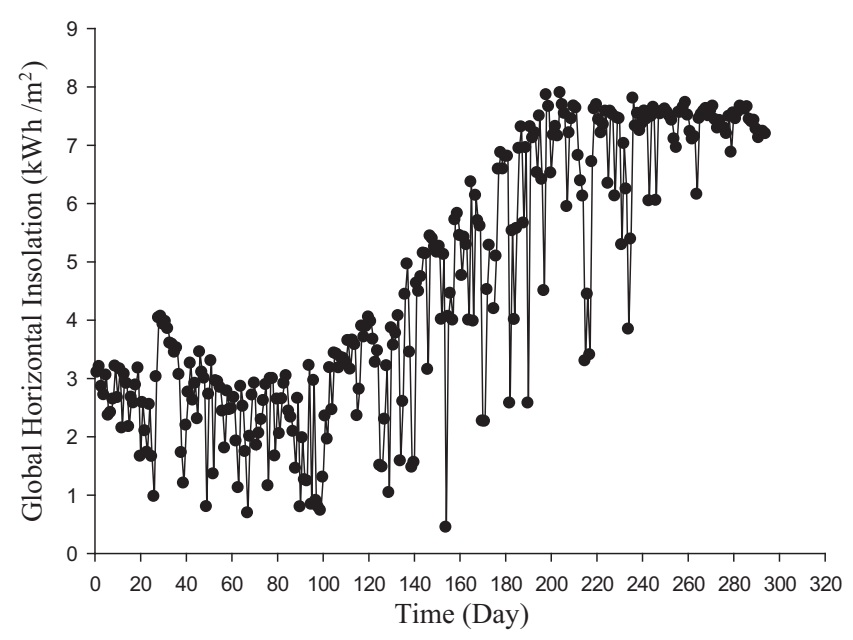

Fig. 7. GHI daily variations.

from day 50 (November 27, 2014) to day 75 (December 22, 2014) followed the ambient temperature variations. As noted before, the temperature difference among the layers dropped starting from day 241 when salinity disturbance was observed (Fig. 3 ). The above observations imply that the temperature difference variations are functions of solar radiation, ambient temperature and salinity gradient. That is, provided the salinity stability, the temperature of all layers strongly follow the variations in ambient temperature, while $\mathrm{GHI}$ is directly correlated with the temperature difference between the layers of the pond.

Fig. 8 shows the variations of average monthly GHI, ambient temperature and solar pond temperatures. Minimal monthly average temperatures for UCZ, NCZ, LCZ and ambient temperature during January 2015 were recorded to be, respectively, 11, 14, 15 and $11^{\circ} \mathrm{C}$. The highest $\mathrm{LCZ}$ temperature obtained was $48^{\circ} \mathrm{C}$ in July 2015 , when the average ambient temperature was $30^{\circ} \mathrm{C}$ and $\mathrm{GHI}$ was $7.4 \mathrm{~kW} \mathrm{~h} / \mathrm{m}^{2}$. This value is in close agreement with the findings of Hassairi et al. (2000) where they reported a maximum temperature of $47^{\circ} \mathrm{C}$ for a GHI of $7.3 \mathrm{~kW} \mathrm{~h} / \mathrm{m}^{2}\left(26.3 \mathrm{MJ} / \mathrm{m}^{2}\right)$ and an ambient temperature of $32^{\circ} \mathrm{C}$ in Tunisia. The pond temperature reached its lowest values when GHI and ambient temperature were both at their lowest values. On the other hand, the pond temperature reached highest values when either the ambient temperature or GHI was at its highest level. That is, during the first month,
October 2014, the ambient temperature was higher than that of March and April, while the trend was opposite for the GHI. Fig. 8 shows that in some periods of the year, the impact of ambient temperature was more significant compared to the GHI in establishing the pond temperatures. For example, although GHI slightly increased from October 2014 to November 2014, the pond temperature decreased in this period of operation due to reduction of the ambient temperature. A similar trend is observed in the duration of December 2014 to January 2015. From January 2015 to February 2015, the pond temperature increased with increasing GHI while the ambient temperature depends remained unchanged. The results presented in Fig. 8 reveal that the pond temperature depends on insolation and ambient temperature which is in agreement with the conclusion made from Figs. 5-7. However, Fig. 8 also indicates that the rate of temperature increase for different pond layers correlates with the ambient temperature even though the GHI increases the pond temperature among the layers particularly in the LCZ. The strong dependency of pond temperature profiles with the ambient temperature variations was also noted by Sakhrieh and Al-Salaymeh (2013). In summary, the presented data show that all layer temperatures follow the trend of ambient temperature variations, and GHI directly affects the temperature gradient within the pond. For example, from January 2015 to April 2015, a sharp increase in the GHI was observed while the pond temperature followed the smooth ambient temperature increase. This could be due to the small pond size that also suffers from the shading effect as well as the possible insufficient thermal insulation. In an effort to minimize the conductive/convective energy losses from the sidewalls and ground, the insulation layer of the pond was improved after about two months of the operation.

Figs. 9 and 10 show hourly temperature variations of the UCZ, NCZ and LCZ, as well as ambient temperature, measured on two consecutive days before (Fig. 9) and after (Fig. 10) the insulation improvement. Note that the temperatures measured on the days after the insulation improvement are lower because of the lower ambient temperature in December. The effect of the proper insulation can be seen in Fig. 6 from the difference between the LCZ and NCZ temperatures. It was observed that under an improved insulation, the pond worked more efficiently and maintained a higher temperature difference between the NCZ and LCZ.

The day-to-night temperature variations of all layers strongly followed the changes in ambient temperature regardless of the insulation level (Figs. 9 and 10) which suggest a significant overnight heat loss through the pond layers to the atmosphere. Therefore, there is the possibility of improving the overall efficiency by minimizing the overnight heat loss such as by providing insulation for the top surface at nights to reduce the convective losses. The significance of this heat loss and the potential source of overnight energy saving is addressed in multiple studies. This conclusion is in agreement with previous finding of Karakilcik et al. (2006). They found that heat loss through the inner surfaces accounted for approximately $85 \%$ of the total heat loss overnight. Bernad et al. (2013) estimated a minimum 85\% total heat loss (inner surface, wall and ground) for a large-scale solar pond during the cold season of the year (October to April). Small ponds will lose a lot more heat from the sides as the surface to volume ratio is higher for small ponds compared to large ponds. Thus, in performance analysis and approximating the thermal efficiency of solar ponds, the size of the pond must be taken into account.

Fig. 11 presents the $\mathrm{GH}$ insolation variations over the years of 2011-2014 recorded at METUNCC. The peak GH insolation was observed in the month of June, on average $7.7 \mathrm{~kW} \mathrm{~h} / \mathrm{m}^{2}$, which is in agreement with 2015 data suggesting lower GH insolation for August and September 2015 compared to that recorded for June $2015\left(7.31 \mathrm{~kW} \mathrm{~h} / \mathrm{m}^{2}\right)$. 


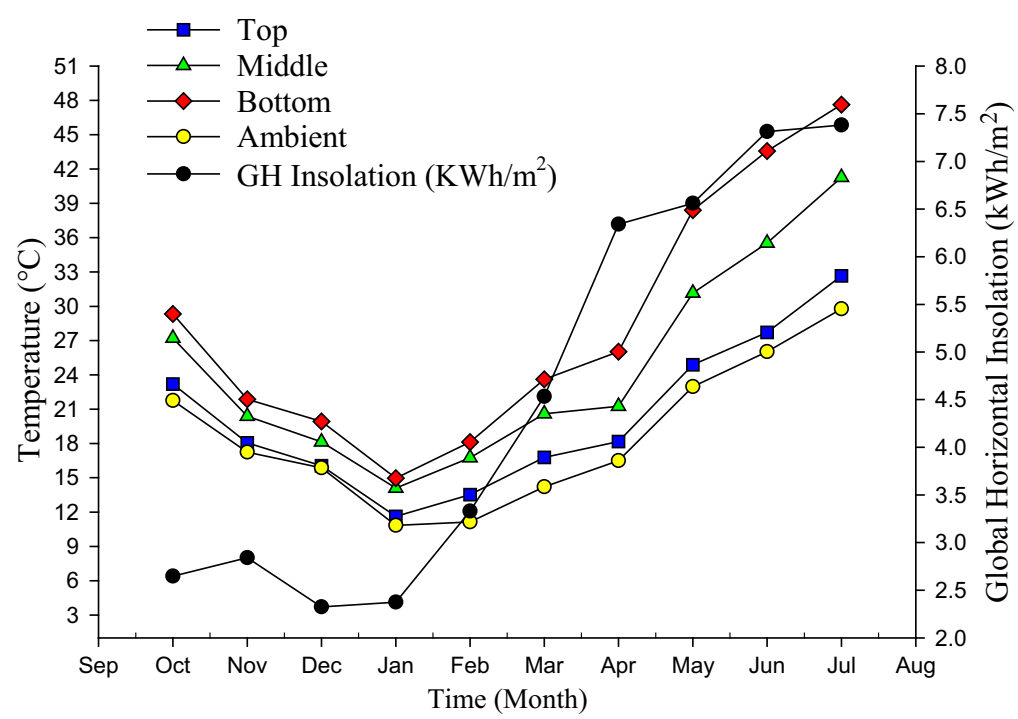

Fig. 8. Average monthly temperature and GHI.

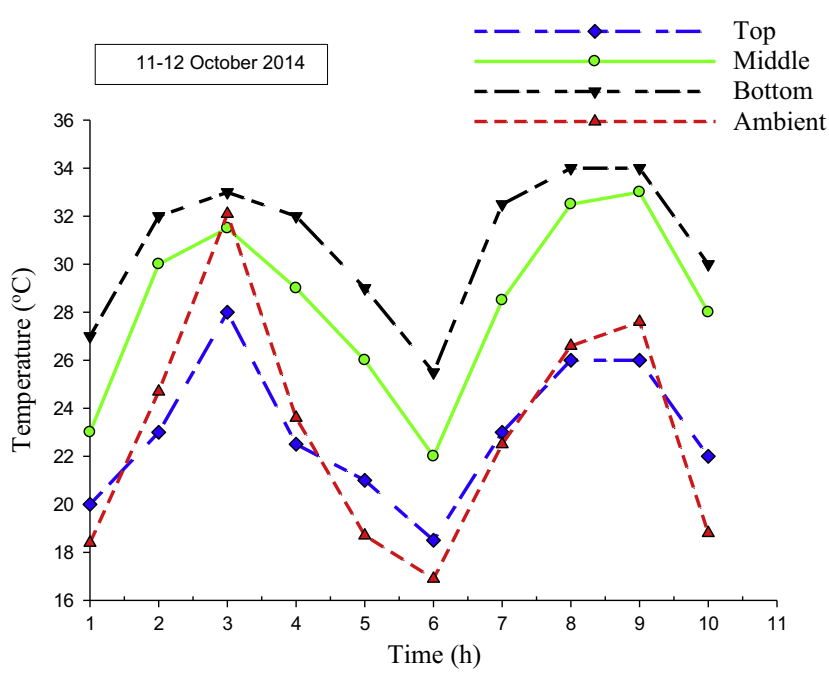

Fig. 9. Day to night hourly temperature of each layer before insulation improvement: For October 11-12, 2014, time as hours 1-10 correspond to 6 a.m., 9 a.m., 1 p. m., 5 p.m., 10 p.m. (October 11) and 6 a.m., 9 a.m., 1 p.m., 5 p.m., 10 p.m. (October $12)$.

Fig. 12 shows the average monthly wind velocity variations, $15 \mathrm{~cm}$ above the solar pond surface as well as the relative humidity $(\mathrm{RH})$ variations during the experimental campaign. As can be seen, the wind velocity showed an increasing trend from October to February, following with a decreasing trend toward May with the second peak in June. Higher wind velocity increases the water mass transfer coefficient above the pond surface, and therefore, it increases the water evaporation rate. In addition, higher wind velocity increases convective heat losses from the pond side walls. It is understood from a simple energy balance around the pond that higher evaporation rate results in lower temperatures in each zone. Evaporation rate was not monitored in this study. Additionally, in this study surface washing was performed such that the level of the water inside the pond was kept constant against the evaporation loss which is again influenced by the wind velocity. In addition, the convective heat losses from the sidewalls were not measured. Thus, assessment of the effect of the wind velocity on the pond performance is not possible.

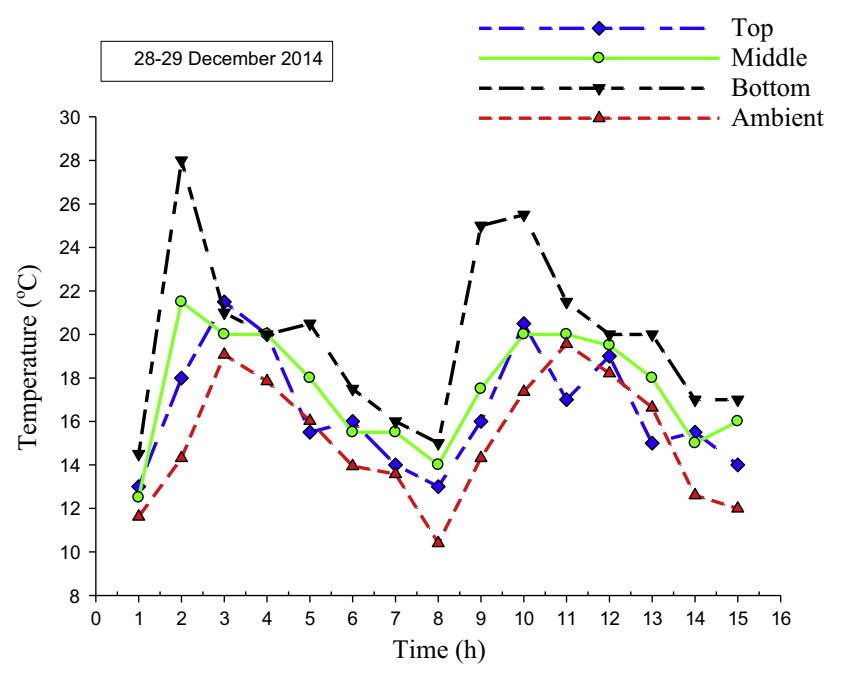

Fig. 10. Day to night hourly temperature of each layer before insulation improvement: For December 28-29, 2014, hours 1-15 correspond to 6 a.m., 9 a.m., 1 p.m., 3 p.m., 5 p.m., 7 p.m., 10 p.m. (December 28 ) and 6 a.m., 9 a.m., 11 a.m., 1 p.m., 4 p. m., 5 p.m., 8 p.m., 10 p.m. (December 29).

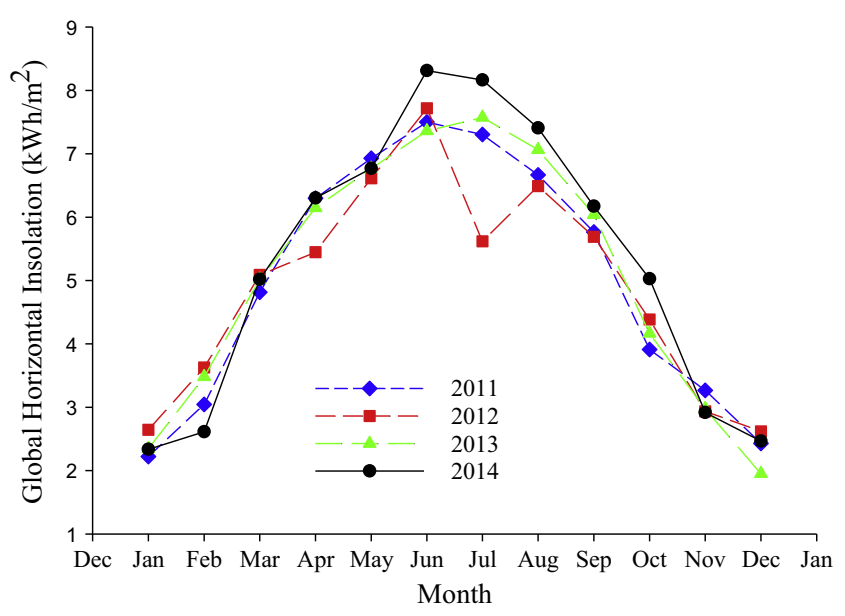

Fig. 11. GHI variations over the years of 2010-2014 recorded at METU NCC. 


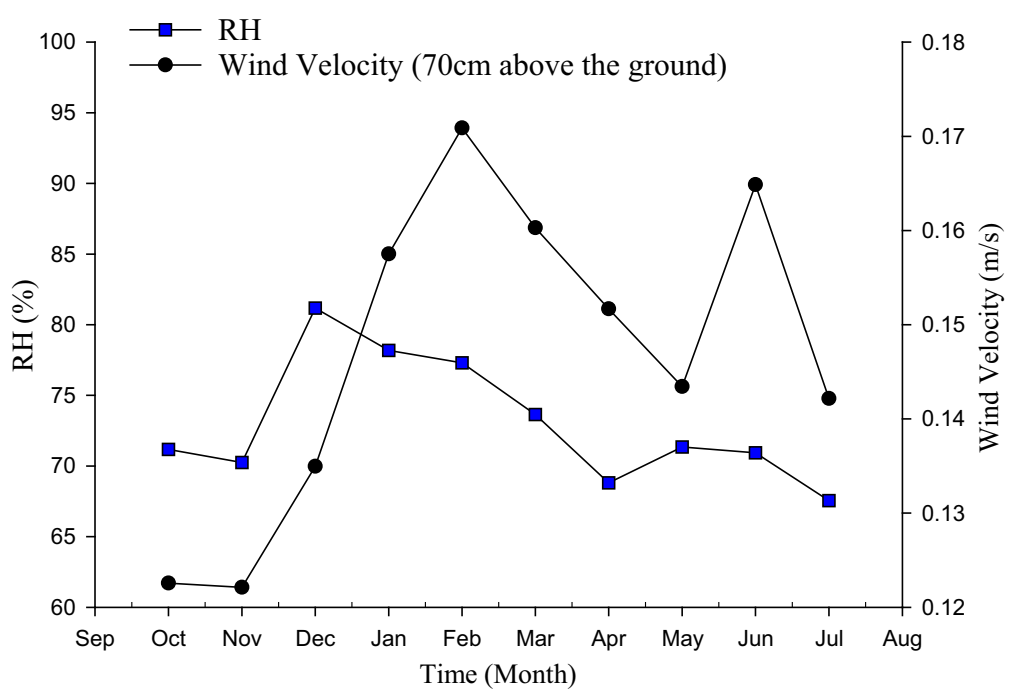

Fig. 12. Relative humidity $(\mathrm{RH})$ and wind velocity variations $(15 \mathrm{~cm}$ above the solar pond surface) during the experimental campain.

The $\mathrm{RH}$ varied from a maximum value (81\%) in December 2014 to a minimum value (67\%) in July 2015 (Fig. 12). Overall, Fig. 12 shows that Northern Cyprus is a humid region. The increased $\mathrm{RH}$ reduces evaporation rate which is in favor of increased pond temperature. Again, since evaporation rates were not quantified, further investigations are required to explore the impact of $\mathrm{RH}$ on the temperature profile of the pond. The presented data in Fig. 12 together with the ambient temperature and insolation data are required for CFD modeling and validations to further predict the degree of influence of each individual parameter on the performance of a salinity gradient solar pond.

The study was subject to several experimental uncertainties including environmental and measurement uncertainties. Fouling and accumulation of dirt in the pond were observed during the course of the experiments that may influence the salinity gradient of the pond. Salinity disturbances may have also occured during raining, sampling and surface washing periods. In particular, fast surface washing may have resulted in some mixing at UCL and $\mathrm{NCZ}$ interface. This phenomenon in fact caused NCZ salinity disturbances at least in one case in the present study. Unexpected raining which is typical in Mediterranean climate could have also changed the UCZ volume necessitating further corrective actions to reduce the level of UCZ. In addition to the environmental uncertainties, monitoring devices and analytical methods were also associated to certain measurement errors. For example, the thermometers had the accuracy of $\pm 0.5^{\circ} \mathrm{C}$. Measurement errors may have also occurred during conductivity measurements and salt analyses as the temperature of the withdrawn samples in the field were higher than the corresponding temperature in the laboratory at the time of conductivity reading, although this temperature effect was corrected.

\section{Conclusions}

The presented experimental investigations provided a daily time resolution for nearly one year of the operation leading to a better understanding of the dynamics of solar ponds. The needs for regular surface washing and undissolved salt for maintaining the stability of the pond were explicitly addressed experimentally to overcome the diffusive and convective disturbances as well as evaporative losses. Ambient temperature, radiation intensity (insolation) and maintaining salinity gradient were found to be the most critical factors in stabilizing the solar pond. The data showed that the temperature of a solar pond follows the variations in ambient temperature while the GH insolation directly correlates with the temperature gradient within the pond. Small disturbances in the salinity gradient adversely affected the performance of the pond due to subsequent convective losses. Salt diffusion rate increased during the summer necessitating more often surface washing. Addition of excess salt to $\mathrm{LCZ}$ at the beginning of the experiment was found to be critical as it would regulate the salt concentration at the saturated level in LCZ. The improved insulation of the pond was observed to generate higher temperature differences between the NCZ and LCZ. The data provided in the present study can be useful for model validation and model improvement for investigating the effect of individual parameter on long term operation of solar ponds.

\section{Acknowledgments}

The authors acknowledge Middle East Technical University Northern Cyprus Campus for providing the financial support to this project. Also, the authors greatly appreciate the generous support provided by Mr. Abdullah Onal for the construction and maintenance of the solar pond. The authors would like to express their appreciations to the department of chemical engineering at Nazarbayev University for their administrative supports.

\section{References}

Bernad, F., Casas, S., Gibert, O., Akbarzadeh, A., Cortina, J.L., Valderrama, C., 2013. Salinity gradient solar pond: validation and simulation model. Sol. Energy 98, 366-374.

Dah, M.O., Ouni, M., Guizani, A., Belghith, A., 2005. Study of temperature and salinity profiles development of solar pond in laboratory. Desalination 183 (1), $179-185$.

El-Sebaii, A.A., Ramadan, M.R.I., Aboul-Enein, S., Khallaf, A.M., 2011. History of the solar ponds: a review study. Renew. Sustain. Energy Rev. 15 (6), 3319-3325.

El-Sebaii, A., Aboul-Enein, S., Ramadan, M., Khallaf, A., 2013. Thermal performance of shallow solar pond under open and closed cycle modes of heat extraction. Sol. Energy 112, 30-41.

Hassairi, M., Safi, M., Chibani, S., 2000. Natural brine solar pond: an experimental study. Sol. Energy 70 (1), 45-50.

Jaefarzadeh, M., 2004. Thermal behavior of a small salinity-gradient solar pond with wall shading effect. Sol. Energy 77 (3), 281-290.

Johnson, H.K., 1999. Simple expressions for correcting wind speed data for elevation. Coast. Eng. 36, 263-269.

Karakilcik, M., Kıymaç, K., Dincer, I., 2006. Experimental and theoretical temperature distributions in a solar pond. Int. J. Heat Mass Transf. 49 (5-6), 825-835.

Karim, C., Slim, Z., Kais, C., Jomâa, S.M., Akbarzadeh, A., 2010. Experimental study of the salt gradient solar pond stability. Sol. Energy 84 (1), 24-31. 
Nie, Z., Bu, L., Zheng, M., Huang, W., 2011. Experimental study of natural brine solar ponds in Tibet. Sol. Energy 85 (7), 1537-1542.

Norton, B., 1992. Solar Energy Thermal Technology. Springer-Verlag, London, pp. $149-159$.

Ouni, M., Guizani, A., Belguith, A., 1998. Simulation of the transient behaviour of salt gradient solar pond in Tunisia. Renew. Energy 14 (1-4), 69-76.

Sadati, S., Qureshi, S.M., Baker, D., 2015. Energetic and economic performance analyses of photovoltaic, parabolic trough collector and wind energy systems for Multan, Pakistan. Renew. Sustain. Energy Rev. 47, 844-855.

Sakhrieh, A., Al-Salaymeh, A., 2013. Experimental and numerical investigations of salt gradient solar pond under Jordanian climate conditions. Energy Convers. Manage. 65, 725-728.

Singh, B., Gomes, J., Tan, L., Date, A., Akbarzadeh, A., 2012. Small scale power generation using low grade heat from solar pond. Proc. Eng., 50-56
Suárez, F., Ruskowitz, J., Childress, A., Tyler, S., 2014. Understanding the expected performance of large-scale solar ponds from laboratory-scale observations and numerical modeling. Appl. Energy 117, 1-10.

Sukhatme, S., Nayak, J., 2008. Principles of thermal collection and storage. J. Chem. Sci. 3, 521-531.

Tabor, H., Matz, R., 1965. A status report on a solar pond project. Sol. Energy 9 (4), $177-182$.

Tabor, H., Weinberger, Z., 1981. Non-convecting solar ponds. Solar Energy Handbook.

Weinberger, H., 1964. The physics of the solar pond. Sol. Energy 8 (2), 45-56.

Zangrando, F., 1980. A simple method to establish salt gradient solar ponds. Sol. Energy 25 (5), 467-470. 